\title{
Montaigne et quelques Italiens: la conversation dans la bibliothèque
}

Jean Balsamo

\section{(2) OpenEdition}

1 Journals

\section{Édition électronique}

URL : http://journals.openedition.org/studifrancesi/5823

DOI : 10.4000/studifrancesi.5823

ISSN : 2421-5856

Éditeur

Rosenberg \& Sellier

\section{Édition imprimée}

Date de publication : 1 mai 2011

Pagination : 3-15

ISSN : 0039-2944

\section{Référence électronique}

Jean Balsamo, « Montaigne et quelques Italiens: la conversation dans la bibliothèque », Studi Francesi [En ligne], 163 (LV | I) | 2011, mis en ligne le 30 novembre 2015, consulté le 09 janvier 2021. URL: http://journals.openedition.org/studifrancesi/5823 ; DOI : https://doi.org/10.4000/studifrancesi.5823

\section{(c)}

Studi Francesi è distribuita con Licenza Creative Commons Attribuzione - Non commerciale - Non opere derivate 4.0 Internazionale. 


\section{Montaigne et quelques Italiens: la conversation dans la bibliothèque}

La bibliothèque, telle que nous la connaissons, est un lieu de silence, d'où la conversation est bannie; c'est un lieu voué au travail, à la lecture silencieuse, et parfois au sommeil. Ce n'est que dans son acception élargie, dans les accès qui conduisent aux salles de lecture, qu'elle permet rencontres et conversations, dans le voisinage immédiat des livres sinon au milieu d'eux. Au cours du XvI ${ }^{\mathrm{e}}$ siècle, la bibliothèque était un des lieux privilégiés des échanges savants et mondains, à travers les deux formes, érudite et princière, qui ont présidé à sa fondation. Dans un cas, la décision politique, en fait une forme d'évergétisme, a conduit à ouvrir des collections de manuscrits rares et d'imprimés à des lettrés désireux de poursuivre des travaux savants parfois sans lien avec la célébration princière; dans l'autre, une collection privée, un cabinet devenu lieu de rencontre de savants et d'écrivains, la bibliothèque monastique étant une des variantes de cette catégorie ${ }^{1}$. Sous cette double forme, la bibliothèque a acquis un statut d'institution, dont le prestige et la notoriété ont fait d'elle un des plus recherchés parmi les admiranda d'une ville ou d'une province. La visite de la «librairie», la rencontre avec le gardien, la conversation avec les lettrés qui la fréquentent deviennent alors un des éléments topiques, au sens géographique et au sens rhétorique, à la fois du voyage, en Italie comme ailleurs en Europe, comme du récit du voyage, à mesure que se codifie ce genre littéraire, point de rencontre des différentes formes érudites. En 1617, dans la révision de la «Tabella delineans in peregrinationibus potissimum observanda» accompagnant son Itinerarium Germaniae, Galliae, Angliae, Italiae, Paul Hentzner indiquait précisément que le voyageur devait aussi visiter les «viros doctos et bibliothecas» ${ }^{2}$. Cette Tabella offrait un plan commode permettant non seulement de concevoir la disposition rhétorique d'un récit de voyage, mais aussi sa topique et son invention, en indiquant les choses à voir et l'ordre de leur description. En 1644, dans son Traité des plus belles bibliothèques, le P. Jacob donna une sorte de guide universel de ce lieu.

La relation des conversations dans la bibliothèque est encore rare dans le Voyage d'Italie (1574-1578) de Nicolas Audebert, premier témoin et explorateur d'un genre inchoatif. Un quart de siècle plus tard, elle est devenue la norme dans le Voyage d'Allemagne de Jacques Esprinchard ${ }^{3}$. À Florence, Audebert notait simplement qu'il «ne

(1) Sur l'histoire de cette institution, voir, outre A.R.A. Hobson, Humanists and Bookbinders, Cambridge, University Press, 1989, Les Humanistes et leurs bibliothèques, éd. R. DE SMET, Louvain-Paris, Sterling, 2002.

(2) P. Hentzner, Itinerarium Germaniae, Galliae, Angliae, Italiae [1592], Wroclaw, Perfert, $1617, \mathrm{f}$. [VIII] $\mathrm{r}$.
(3) J. EsPRINCHARD, Journal des voyages en diverses contrées de l'Europe, La Rochelle, Bibliothèque municipale, ms 4 [rédigé avant 1604]; L. CHATENAY, Vie de Jacques Esprinchard Rochelais et Journal de ses voyages au XVI siècle, Paris, SEVPN, 1957; voir notre étude Jacques Esprinchard en Allemagne: aux origines du voyage savant (1597-1598), «Romanic Review», 94, 2003, 1-2, pp. 26-42. 
fault oublier à veoir l'excellente bibliothèque», sans plus de détails. C'est au palais ducal en fait qu'il vit des livres, grâce à l'entremise de Pietro Vettori, et chez Vettori lui-même, qui lui fit les honneurs de sa «belle bibliothèque», où il lui montra:

Un vieil contrat escript en escorce d'arbre, mais fort examiné d'ancienneté et lequel estoit d'une lettre antique fort difficile à lire ${ }^{4}$.

C'est autour de cet objet que tourna la conversation, en forme d'expertise savante. À sa manière, dans le développement du lieu rhétorique de la bibliothèque, le livre «escript en escorce d'arbre», conjuguant l'objet de curiosité au prétexte érudit, allait jouer un rôle particulier. Au mois de février 1586, Arnold van Buchel, un étudiant originaire d'Utrecht, au cours de sa peregrinatio academica, visita la bibliothèque de l'Abbaye de Saint-Germain-des-Prés à Paris. Il fit de cette visite une relation détaillée dans son journal et nota en particulier des curiosités et quelques trésors au milieu de la saleté du lieu'. Il remarqua en particulier «un livre écrit sur bois ou sur une écorce très mince». Quelques semaines plus tôt, en compagnie de Louis Carrion, de Bruges, qui l'avait introduit dans les milieux lettrés de la capitale, il avait rendu visite à François de La Croix du Maine, «grand ami des lettres et grand chercheur de livres», qui reçut ses visiteurs dans sa bibliothèque, «riche en ouvrages de tout genre». Van Buchel donna un aperçu des livres, mais ne transcrivit pas les propos échangés, et il nous faut faire un effort d'imagination pour reconstituer cette conversation qui était un véritable rituel savant: le collectionneur donnant à voir des objets en les commentant, le visiteur prenant soin de noter dans sa mémoire ce qui lui était montré, afin de le retranscrire dans son journal. Cette conversation en fait était limitée à des objets et à un usage lettré.

La visite que fit Montaigne, le 6 mars 1581, à la Bibliothèque Vaticane, et qu'il relata dans son journal, est sans doute la plus célèbre de ces visites et de ces «conversations dans la bibliothèque» ${ }^{6}$. D'une certaine manière, elle ordonne le récit en lui conférant son véritable climax. Le secrétaire, qui avait tenu la première partie du journal, avait noté les premières étapes de ce rituel savant, à Meaux, où Montaigne «fut visiter le Thresorier de l'Eglise Saint-Etienne, nommé Juste Terrelle [...] qui lui montra sa librairie» ${ }^{7}$, à Neufchâteau, où il se rendit en l'église des Cordeliers pour voir «leur librairie où il y a force livres, mais rien de rare» ${ }^{8}$, à Bâle. La visite était alors liée à la notoriété d'un savant, elle était animée par une forme de curiosité pour la rareté, qui fera le fond de la conversation in situ. À Rome, Montaigne, qui avait donné congé à son secrétaire, fit lui-même le récit de sa visite. Il décrit le lieu avec précision, indique sa disposition, son ordonnance, la présentation des livres, et il dresse une sorte d'inventaire des curiosités les plus notables qui lui sont montrées, parmi lesquelles précisément «un livre de Chine, le caractère sauvage, les feuilles de certaine matière beaucoup plus tendre et pellucide que nostre papier», écrit sur «la

(4) N. Audebert, Voyage d'Italie, éd. A. Olivero, Rome, Lucarini Editore, 1981, I, p. 257.

(5) A. VAN BUCHEL, Commentarius rerum quotidianarum [1584-1585], Utrecht, Rijksuniversiteit, Algemene Bibliothek, ms 798 (Hist. 132, 133), t. I (France). Le manuscrit a fait l'objet d'une traduction pour la partie française par A. VIDIER, Description de Paris par Arnold van Buchel d'Utrecht, « Mémoires de la Société de Paris et de l'Île-de-France », XXVI, 1899, pp. 59-184; ici, p. 149.

(6) Voir F. Caldari Bevilacqua, Montaigne alla
Biblioteca Vaticana. Montaigne e l'Italia, éd. E. BALMAS, Genève, Slatkine, 1985, pp. 363-390, ainsi que W. BOuTCHER, 'Le moyen de voir ce Senecque escrit à la main': Montaigne's Journal de voyage and the Politics of Science and Faveur in the Vatican Library «Michigan Romance Studies»: (Re)interprétations: études sur le seizième siècle, éd. J. O’BrIEN, 1995, pp. 177-214.

(7) Montaigne, Journal de voyage, éd. F. RigoLOT, Paris, PUF, 1992, p. 4.

(8) Ibid., p. 8. 
membrane de quelque arbre» ${ }^{9}$. Le récit de Montaigne est organisé suivant une description, complétée d'un catalogue:

Je fus voir la librairie du Vatican, qui est en cinq ou six salles tout de suite. Il y a un grand nombre de livres [...] Item une Bible imprimée en parchemin, de celles que Plantin vient de faire en quatre langues, laquelle le Roy Philippe a envoyée à ce Pape comme il dit en l'inscription de la relieure; l'original du livre que le Roy d'Angleterre composa contre Luther, lequel il envoya, il y a environ cinquante ans, au Pape Leon dixiesme.

Cette description repose en fait sur un ensemble de «lieux» que l'on retrouve développés par d'autres visiteurs. Ainsi, en janvier 1589, un autre gentilhomme français pouvait-il écrire:

J'ay veu l'antienne bibliotheque en laquelle il y a six chambres, toutes pleines de livres, quasi tous escritz à la main; entre les livres l'on nous a monstré [...] Henri d'Angleterre contre Martin Luther, envoyé au pape [...] la Bible envoyée au pape par le roy d'Espagne: elle est en parchemin et la suscription dessus ${ }^{10}$.

Les circonstances de la visite sont évoquées sommairement par Montaigne. La visite semble, en apparence du moins, relever d'une simple initiative personnelle, facilitée par un usage établi, quotidien, comme si la librairie était ouverte à tout un chacun, sans distinction ni préséance: nulle tractation n'est rapportée, nulle démarche pour accéder à la bibliothèque, nulle discussion en cours de visite, sinon avec un gentilhomme anonyme, qui accompagne partout le visiteur et le convie «d'en user quand [il] voudrai[t]». Des comparses apparaissent toutefois, mais comme en creux, par le jeu de la voix passive («des coffres qui me furent tous ouverts»), ou moins indirectement, sous la forme du sujet anonyme qui donne des explications au visiteur («Ils tiennent que [...] ils tiennent»). Au milieu de son récit toutefois, Montaigne est conduit à relativiser les conditions de la visite et la facilité de l'accès, par l'exemple contraire de l'ambassadeur de France, Louis Chasteigner Abain de La Rocheposay ${ }^{11}$. Ce dernier en effet n'avait jamais pu obtenir d'accéder à ces lieux et de voir enfin «ce Seneque escrit à la main, ce qu'il desiroit infiniment», et Montaigne, se fondant sur son échec, pouvait s'étonner avec une feinte naïveté d'avoir été admis si facilement, attribuant à la fortune le privilège qui lui avait été fait, non pas celui de visiter la bibliothèque, où d'autres voyageurs pénétraient sans difficulté, mais celui de ne pas avoir subi la même mésaventure que La Rocheposay et de s'être vu refuser son accès ${ }^{12}$.

On a voulu expliquer la différence de traitement comme la conséquence d'une différence de «pratique» dans la maîtrise des codes et de l'étiquette, excellente chez Montaigne, insuffisante chez le diplomate malheureux ${ }^{13}$. L'ambassadeur en effet avait confié à Montaigne que pour être autorisé à visiter la Bibliothèque Vaticane, il aurait

(9) Ibid., pp. 111-113.

(10) Voyage de Provence et d'Italie (ms fr. 5550 B.N. Paris), éd. L. Monga, «Biblioteca del viaggio in Italia», 49, Genève, Slatkine, 1994, p. 73.

(11) Sur le personnage et ses relations avec Montaigne, voir P. DESAN, L'appel de Rome, ou comment Montaigne ne devint jamais ambassadeur Chemins de l'exil. Havres de paix, actes du colloque, Tours, CESR, 2007, éd. J. Balsamo et C. Lastraioli, Paris, Champion, 2010, pp. 229-259.

(12) L'érudit Audebert ne mentionne pas une telle visite. Nicolas BÉNARD en revanche la décrit sommairement, Voyage de Hierusalem ensemble son retour par l'Italie, Paris, D. Moreau, 1621, p. 434. Peiresc pour sa part entretint une abondante correspondance avec les bibliothécaires, voir M. CERESA, Peiresc e la Biblioteca Vaticana Peiresc et l'Italie, dir. M. Fumaroli, Paris, Baudry, 2009, pp. 265-277.

(13) Sur cette question, voir l'analyse de X. LE Person, Montaigne et les 'practiques' politiques de son temps Montaigne politique, éd. P. Desan, Paris, H. Champion, 2006, pp. 95-112. 
dû «faire la cour au cardinal Charlet [Sirleto], maistre de cette librairie». Peut-on croire pour autant, comme le suggère la critique, qu'il accordait de l'importance à seulement voir un livre et que son échec dans ce projet de nature privé était dû à une question d'amour-propre qui l'aurait dissuadé de faire la démarche nécessaire à cette fin? Il ne s'agissait pas en fait d'une affaire personnelle mais d'une opposition politique. Une telle démarche était impossible à concevoir dans le cadre des relations diplomatiques et des rapports de pouvoir au sein de la Cour de Rome, entre le parti français et le parti espagnol de la Curie que représentait le cardinal Sirleto. La faveur que La Rocheposay ne voulait pas solliciter était d'autant plus ostensiblement accordée à Montaigne; elle procédait d'une décision discrétionnaire destinée à flatter l'un pour mieux humilier l'autre, à récompenser et à châtier. Par le seul fait qu'elle aurait pu lui être refusée, la visite de la Bibliothèque Vaticane, dans son apparente banalité, était la première d'une série de distinctions octroyées à Montaigne, précédant l'octroi de la citoyenneté romaine et la censure favorable des Essais, avant la conversation avec le Maestro del Sacro Palazzo ${ }^{14}$. Mais quelques semaines plus tard, à Urbin, Montaigne chercha vainement à visiter la «belle librairie» du duc d'Urbin, dont on ne trouva plus la clé. Ce refus, caché sous un mauvais prétexte, n'était pas, là non plus, d'ordre personnel, il était opposé à un gentilhomme français, à un sujet du roi de France dans une cour qui lui était hostile; Montaigne en comprit parfaitement le sens: «Ils ont l'inclination Espagnole. Les armes du Roy d'Espaigne se voyent en rang de faveur, et l'Ordre d'Angleterre et de la Toison d'Espaigne, et rien du nostre» ${ }^{15}$.

Montaigne possédait lui-même une bibliothèque, «une des belles entre les librairies de village» ${ }^{16}$, qu'il avait fait aménager dans la tour principale de son château. Il la décrit en détail dans un passage longuement amplifié et corrigé dans la dernière rédaction des Essais:

La figure en est ronde, et n'a de plat que ce qu'il faut à ma table et à mon siège: et vient m'offrant en se courbant, d'une veue, tous mes livres, rengez sur des pupiltres à cinq degrez tout à l'environ. Elle a trois veues de riche et libre prospect, et seize pas de vuide en diame$\operatorname{tre}^{17}$.

Cette présentation suit la disposition et l'ordonnance des lieux. La librairie se trouve $\mathrm{au}$, troisième niveau du bâtiment, en fait le deuxième étage, au dessus de la chapelle,

(14) Cette question a été entièrement renouvelée par un ensemble de documents récemment découvert; voir A. LEGROS, Montaigne face à ses censeurs romains de 1581 (Mise à jour), «Bibliothèque d'Humanisme et Renaissance», 71, 2009, p. 7-34.

(15) Journal, p. 148. Sur cet épisode, voir W. Boutcher, Michel de Montaigne e "Frédéric Maria de la Rovere": la chiave nascosta della biblioteca dell'ultimo duca d'Urbino I Della Rovere nell'Italia delle Corti, vol. III, Cultura e letteratura, éd. B. CLERI et alii, Urbino, Quattroventi, 2002, pp. 93-114.

(16) Les Essais, II, 17, éd. J. Balsamo, M. Magnien, C. Magnien-Simonin, Paris, Gallimard, 2007 («Bibliothèque de la Pléiade»), p. 689.

(17) Ibid., III, 3, pp. 869-870. Le texte de l'Exemplaire de Bordeaux propose un premier état de l'ajout, très lacunaire. Il est le résultat d'une rédaction en deux moments et d'une disposition graphique imposée par un défaut du papier au coin inférieur droit du feuillet 5A2R: une première partie (jusqu'à confuse) a été transcrite par Montaigne dans la marge latérale droite à partir d'un brouillon préalable; elle porte quelques rares corrections postérieures, peut-être contemporaines de la seconde partie (miserable [...] estre), rédigée dans la marge du bas. Cette seconde partie semble avoir été directement élaborée sur l'Exemplaire. L'édition posthume, établie sur la mise au net d'un exemplaire de travail différent de l'Exemplaire de Bordeaux, donne le texte complet avec trois variantes importantes: l'une reprend une leçon modifiée sur EB $\left(j^{\prime} y\right)$; la deuxième modifie la rédaction «Mon esprit ne va pas si les iambes ne l'agitent» et lui donne un sens différent: «Mon esprit ne va pas seul, comme si les jambes l'agitent»; là où EB portait «mes liures rengez a cinq degrez tout a l'enuiron», la troisième apporte une précision capitale concernant la disposition des livres, «sur des pulpitres», précision qui ne peut en aucun cas être une interpolation de Marie de Gournay, celle-ci n'ayant pas alors visité la bibliothèque. 
de plain-pied, et de la chambre où Montaigne couche seul, et qu'il céda au roi de Navarre lors de la visite que celui-ci lui fit en 1584. Une garde-robe est au dernier étage. La librairie est prolongée, au même niveau, par un cabinet. La distinction entre ces deux pièces est ici fonctionnelle. Le cabinet est un espace réduit, qu'une cheminée permet de chauffer en hiver; il est comme le lieu le plus intime de la librairie considérée dans son ensemble. Sa décoration confirme l'attention que Montaigne portait à ce lieu, non seulement en termes de confort, mais surtout en termes symboliques. Le cabinet en effet, introduit dans les demeures royales au début du siècle, confirme un decorum aristocratique ${ }^{18}$. Celui de Montaigne est «poly», orné de peintures et d'inscriptions, qui ont pu être partiellement déchiffrées; elles renvoient à la vie de Montaigne, et les grotesques qui les accompagnaient, à la genèse même des Essais ${ }^{19}$.

La librairie proprement dite a un décor réduit à deux éléments principaux: d'une part les ouvertures sur le paysage, trois «veues de riche et libre prospect»; d'autre part, l'ordonnance des livres que Montaigne assis voit autour de lui, «mille livres» ${ }^{20}$, ainsi qu'il l'écrit ailleurs, «rangez sur des pulpitres à cinq degrez». La plupart d'entre eux devaient être rangés sur des étagères, selon l'usage qui s'est imposé à la fin du XVI siècle. Les volumes provenant de cette bibliothèque qui nous ont été conservés dans leur première reliure portent en effet des titres calligraphiés au dos. On ignore leur mode de classement. Certaines formules du chapitre «Des livres» suggère une répartition par genres ${ }^{21}$. Il est possible toutefois qu'en plus de ces étagères, un pupitre au sens moderne du terme, une tablette de lecture, inclinée à hauteur d'appui, ait entouré la pièce, plus pour porter les livres en cours de consultation que pour l'ostentation de volumes précieux. Montaigne ne mentionne pas la décoration de la librairie, au contraire de celle du cabinet, et il ne fait aucune allusion en particulier aux célèbres «poutres peintes», qui faisaient de ce lieu une véritable «bibliothèque parlante» en adaptant sur un mode provincial, sans autres exemples connus, marqué par la rusticité des poutres, le modèle humaniste des sentences peintes sur des murs ${ }^{22}$.

À travers la description qu'en donne Montaigne, la bibliothèque apparaît comme un lieu à forte charge symbolique dont il convient de déchiffrer dans leur cohérence une suite d'éléments en apparence contradictoires, destinés en fait à l'ostentation d'un statut social, et une référence culturelle présentée comme un art de vivre. La bibliothèque, en tant que lieu, renvoie précisément, fût-ce sous forme allusive, à un modèle architectural princier par rapport auquel le gentilhomme Montaigne cherche à se situer. Nous savons quelle était sa connaissance de l'architecture de son temps et l'usage qu'il en faisait dans un discours moral et politique ${ }^{23}$. En tant que telle toutefois, la librairie n'avait pas encore, en France du moins, de réalité architecturale ni de codification précise. Elle était généralement située dans un lieu commode, adapté à cet usage particulier. A Fontainebleau, la librairie du roi se trouvait au-dessus de la galerie. Or Montaigne parle précisément de la galerie qu'il aurait pu faire élever sur

(18) Sur le cabinet, voir M. Chatenet, La Cour de France au XVI siècle. Vie sociale et architecture, Paris, Picard, 2002, pp. 152-153.

(19) Voir A. LEgROS, Travail de deuil et art de vivre: les deux inscriptions votives de la tour de Montaigne, «Montaigne Studies», XI, 1999, pp. 137-154.

(20) Les Essais, III, 12, p. 1103.

(21) Voir Michel de Montaigne et son temps. Collection Francis Pottiée-Sperry, Paris, Sotheby's, 27 novembre 2003 , p. 84 , n. 80 . Sur la disposition des livres dans les bibliothèques de la Renaissance, voir T. Kimball BROOKER, Upright Works: the Emergence of the Vertical Library in the Sixteenth Century, thè- se, The University of Chicago, 1996, et, du même auteur, Bindings commissioned for Francis I's 'Italian library' with horizontal spine titles dating from the late 1530 s to 1540 , «Bulletin du Bibliophile», 1997, 1, pp. 33-91.

(22) Voir A. Legros, Essais sur poutres. Peintures et inscriptions chez Montaigne, Paris, Klincksieck, 2000; les sentences peintes ont fait l'objet d'une édition critique, Les Essais, éd. citée, pp. 1311-1318.

(23) Voir notre étude Des Pyramides d'Egypte au phare de Cordouan: Montaigne, l'architecture et le temps «Littérature et architecture», éd. L. RICHER, Lyon, CEDIC, 2004, pp. 9-19. 
un mur pour la «coudre» à sa bibliothèque: elle aurait eu cent pas de long et douze de large, et il aurait pu déambuler à loisir, comme en un «proumenoir». Sous une forme négative, la belle galerie restant de l'ordre du regret, il fait ici une claire référence au modèle prestigieux de l'architecture royale, et il témoigne qu'il sait parfaitement ce que représente la galerie: celle-ci est un lieu privé, et non pas public, où se met en scène pour quelques intimes la personne privée du souverain ${ }^{24}$. Dans la seconde moi-

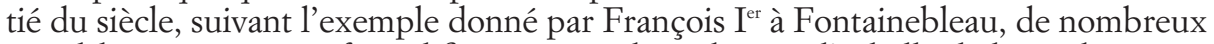
gentilshommes avaient fait édifier et orner des galeries à l'échelle de leurs demeures en province. En renonçant lui-même à un tel projet, Montaigne cédait peut-être à des impératifs financiers et à des dépenses, incompatibles avec la situation dans laquelle il se trouvait au moment des guerres civiles ou après la mairie de Bordeaux. Il avance également le prétexte du soin et du souci qu'une telle construction lui aurait coûtés. Il exprime surtout, à sa manière, un refus. Il ne rejette certes pas le modèle royal en tant que tel, qu'il continue d'admirer et de respecter, lié par une obéissance qui le confirme dans son rang nobiliaire; il refuse de suivre ce modèle de cour et d'y être soumis chez lui, en un lieu désormais voué à la retraite et à la solitude, en un lieu où il entend se soustraire à toute «communauté» et à toute «domination». Le désir de retraite et d'autonomie est devenu si fort en lui qu'il peut considérer comme aliénant de bâtir une galerie et d'imiter une forme d'architecture royale qui, tout en étant une forme privée, ressortirait encore à une forme d'obligation civile, liée à des negotia. La bibliothèque telle que la conçoit Montaigne, n'est pas seulement le lieu où vivre sa solitude, mais le lieu même, à l'intérieur du château, marquant les limites qui séparent les negotia de l'otium, le lieu où cette opposition est constamment mise en évidence. Dans son château, Montaigne vit encore en société; il impose son autorité seigneuriale sur son «mesnage», il confirme son rang, il reçoit ses hôtes et vit avec sa famille. Dans sa librairie, il s'en détourne et fait de la solitude non plus le double positif d'une activité nécessaire, mais l'activité par excellence, qui finit par occuper toute sa vie et se confondre avec elle. La bibliothèque est le lieu ultime de la solitude complète, acceptée et assumée, sinon entièrement voulue, d'une solitude silencieuse.

Or c'est en relation à autrui et à la vie sociale que Montaigne évoque ce lieu. Il décrit sa bibliothèque dans un chapitre consacré aux trois «commerces» ou aux «conversations», dans le sens large de fréquentation, dans lequel il traite précisément des relations choisies, fondées sur l'échange et la parole, auxquelles il aime se dédier, ou du moins qu'il a aimées, et qu'il distingue des relations civiles, dues «au monde par obligation»: la compagnie des hommes habiles et doctes et leurs entretiens, le commerce des dames et la galanterie et, s'ajoutant à la fréquentation des personnes, celle des livres ${ }^{25}$. Ces trois fréquentations, réunies en une même argumentation, sont habituellement présentées par la critique comme le triptyque harmonieux de la sociabilité idéale que Montaigne édifie à travers un art de la parole privée, qui reprend et renouvelle le modèle donné par les théoriciens italiens de la civilité, Stefano Guazzo en particulier, dont la Civil conversazione, publiée en 1574, connaissait un succès européen $^{26}$. A l'exemple de Guazzo, Montaigne trace en effet le cadre de la

(24) Voir J. GuIllaume, La galerie dans le château français: place et fonctions, «Revue de l'art», 102, 1993, pp. 32-42. Sur Fontainebleau, Fr. Boudon et J. BléCON, Le Château de Fontainebleau de François $I^{e r}$ à Henri IV, Paris, Picard, 1998.

(25) Sur ce chapitre, voir G. NorTon, De trois commerces and Montaigne's populous solitude, «French Review», 3, 1970, pp. 101-109.
(26) Sur la complexité de la relation de Montaigne à la Civil conversazione, voir M. Tetel, Présences italiennes dans les Essais de Montaigne, Paris, $\mathrm{H}$. Champion, 1992, pp. 12-27, ainsi que N. PANICHI, 'Filosofare conversando': Montaigne lettore di Stefano Guazzo, «Studi umanistici piceni», 18, 1998, pp. 115-128. 
conversation privée, en définissant ses interlocuteurs et en examinant les conditions de sa réalisation et de sa perfection. Dans la suite du livre III des Essais, dans des développements complémentaires qui prolongent différentes perspectives ouvertes dans les deux premiers livres, il examine en termes critiques les formes des relations privées comme celles des relations civiles et politiques, avec le prince, les Grands, les institutions. Il met ainsi en lumière leurs aspects particuliers et pour ainsi dire techniques, rhétoriques et argumentatifs, mais aussi leur condition éthique dans le discours de «diversion», le discours politique, les formes du conseil au prince, le discours familial, l'esthétique du débat. Le chapitre 5 «Sur des vers de Virgile», dans lequel Montaigne commente sur un mode galant un passage de l'Enéide (VIII, 387-392) célébré par Ronsard, est un exemple longuement amplifié de la conversation avec les Dames. Cette typologie des conversations et de leurs modes constitue à sa manière une subtile variation des formes de parole publique et privée dont Guazzo avait donné le canon. Dans le chapitre «De trois commerces», Montaigne propose bien, en apparence du moins, un développement original du premier thème porté sur le titre même de l'ouvrage italien: «Si tratta in generale de' frutti che si cavano dal conversare». La première fréquentation, celle des hommes doctes, régie par les règles de l'amitié, éclairée par la vérité, est la plus exquise; celle des Dames «belles et honnestes» fondée sur le désir et illuminée par la beauté, repose sur une relation de séduction qui exige une économie savamment réglée, capable de tenir la balance entre la passion et la dissimulation des sentiments. Ces deux conversations ont leurs lieux: pour la première, toutes les occasions où se rencontrent les honnêtes hommes, en voyage comme à la cour; pour la seconde, l'intimité est requise, celle du cabinet des Dames, où elles peuvent offrir leur «commerce un peu privé» ${ }^{27}$. C'est cette intimité que Montaigne évoquait, dès 1580, dans le chapitre dédié à une de ses protectrices, la comtesse de Guiche, Corisande d'Andoins, à qui il offrait un recueil de sonnets amoureux de La Boétie et à qui il promettait de dire «un jour à l'oreille» la «belle et noble ardeur» qui brûlait son austère $\mathrm{ami}^{28}$.

À la différence de la Civil conversazione toutefois, la typologie élaborée par Montaigne dans «De trois commerces» n'a rien d'un programme et elle ne cherche pas à enseigner. Montaigne ne fait pas la théorie de la parole en société. Son choix se donne d'emblée et se confirme, à mesure qu'il s'énonce, sur le mode de la difficulté, de la nostalgie et du regret, comme l'échec et la fin des conversations privées, des amitiés savantes et de la galanterie, également exquises. Montaigne prétend même être incapable de se plier aux conversations familières, dont la banalité émousse ses facultés. Son tempérament, sa «complexion difficile» le rend «délicat à la pratique des hommes» en général:

Peu d'entretiens doncq m'arrestent sans vigueur et sans effort [...] il m'advient souvent, en telle sorte de propos abattus et lasches, propos de contenance, de dire et respondre des songes et bestises indignes d'un enfant, et ridicules: ou de me tenir en silence, plus ineptement encore et incivilement ${ }^{29}$.

Les défauts mêmes de sa parole lui interdisent toute véritable conversation. Montaigne ne sait plus parler. Sa parole manque de convenance; il est trop concis, trop recherché, trop spirituel pour ses interlocuteurs; il est toujours pris par la tentation de «favellar in punta di forchetta». Montaigne ne met pas en évidence son goût pour une 
conversation conçue comme une subtile escrime mondaine, il déplore l'agressivité de son mode de parle. Cette formule en langue italienne a souvent été mal comprise, parce que son origine et son contexte n'ont pas été identifiés. Montaigne la tire en fait de l'Hercolano de Benedetto Varchi, et d'un passage satirique, où elle a un sens clairement péjoratif:

Di coloro, che favellano in punta di forchetta, cioè troppo squisitamente, e affetatamente, e (come si dice hoggi), per quici, et quindi, si dice andare sù per le cime degli alberi simile a quello, cercare de' fichi in vetta ${ }^{30}$.

Mais si cette expression renvoie bien à la trattatistica italienne et si elle confirme non seulement la connaissance parfaite que Montaigne avait de l'art de parler mais aussi l'ampleur et la diversité de ses lectures italiennes ${ }^{31}$, le chapitre «De trois commerces» ne propose pas une civile conversation à la française; il en est au contraire la critique radicale et la réfutation, qui conclut à l'impossibilité même de toute conversation. La véritable conversation ne peut se déployer que dans une relation d'amitié parfaite, où l'âme peut mettre au jour toutes ses ressources les plus exquises. Or la relation des amis est rare à la mesure de sa perfection; celle des «belles et honnestes Dames» est soumise au passage du temps et «se flestrit avec l'aage». Ces conversations ont eu leur temps et Montaigne en déplore la fin. Les autres conversations, privées ou publiques sont aliénantes; toutes sont bornées et fragiles, ces «commerces» sont «despendants d'autruy», incompatibles avec l'autonomie de celui qui ne veut dépendre de personne. C'est dans cette situation de déshérence que prend sens, comme un pis-aller, le «commerce» avec les livres, dans la librairie. Mais contrairement aux deux autres «commerces», il s'agit d'une simple fréquentation, au sens large du terme, qui n'implique pas d'échange de paroles.

La conversation avec les livres est une «conversation» silencieuse, au sein de la solitude ${ }^{32}$. Il n'y a dans cette formulation aucun paradoxe, il ne s'agit pas d'un oxymore, mais plutôt d'une figure d'ironie, par laquelle Montaigne renverse un grand «lieu» de la culture européenne depuis l'Antiquité, dont il mettait en évidence le fondement métaphorique. Dans un autre chapitre des Essais, dans un passage rédigé douze sinon quinze ans plus tôt, Montaigne évoquait une première conversation avec ses livres:

Quelque langue que parlent mes livres, je leur parle en la mienne ${ }^{33}$.

Cette conversation était conçue comme un échange de deux paroles, par la lecture et l'annotation. Montaigne présentait alors en détail ses modes de lecture, il faisait allusion aux annotations dont il truffait les marges de ses livres et les notes de synthèse qui ouvraient ou concluaient son César, son Guichardin, son exemplaire des Mémoires de Guillaume et Martin du Bellay. On conserve quelques volumes lui ayant

(30) Benedetto Varchi, Hercolano, Venise, Giunti, 1570, p. 82; P. Villey avait pourtant indiqué l'ouvrage de Varchi comme une source des Essais et noté que Montaigne en avait tiré plusieurs références pour l'Apologie de Raymond Sebond, dont trois vers de Dante. Il n'avait pas identifié l'origine de la formule «favellar in punta di forchetta», Les Sources et l'évolution des Essais de Montaigne, Paris, Hachette, 1933, t. I, pp. 261-263.

(31) F. GiaconE vient de mettre en lumière une source italienne de Montaigne, jusqu'ici non iden- tifiée, la tragédie Giocasta, de Lodovico Dolce, La Source du vers 'che ricordarsi il ben doppia la noia' de Michel de Montaigne La Langue de Rabelais, la langue de Montaigne, actes du colloque, Rome 2003, Genève, Droz, 2009, pp. 587-592.

(32) Voir D. MÉnAGER, Montaigne, la 'librairie' et le 'pays sauvage' Études seiziémistes offertes à Monsieur le Professeur V.-L. Saulnier, Genève, Droz, 1980 , pp. 383-394.

(33) Les Essais, II, 10, p. 440. 
appartenu. Ils confirment à leur manière une telle conversation, en français. Nous savons aussi qu'à une époque plus ancienne, Montaigne parlait à ses livres en leur propre langue, en latin et parfois même en $\operatorname{grec}^{34}$. En 1564, il avait lu attentivement le De natura rerum de Lucrèce et l'avait annoté en latin ${ }^{35}$. Mais de même que la langue de ses annotations avait changé, son rapport aux livres lui-même avait changé. Sur les premiers volumes qu'il avait acquis, Montaigne avait porté, accolée à son nom, la mention «et amicorum»; il imitait alors un modèle humaniste dont Jean Grolier avait donné la plus célèbre illustration bibliophilique ${ }^{36}$. Ces premiers volumes étaient le témoignage de liens implicites confirmant une conversation universitaire et civile. Très tôt pourtant Montaigne abandonna cette formule, qui ne figure plus avec la mention de possession portée sur le Lucrèce.

En évoquant son «commerce» avec les livres, Montaigne se représente en lecteur indifférent et nonchalant: «là je feuillette à cette heure un livre, à cette heure un autre» ${ }^{37}$. Cette représentation est contredite par ce que nous connaissons des pratiques effectives de Montaigne en tant que lecteur, un lecteur attentif, cultivé, ayant reçu une excellente formation philologique de son maître Turnèbe et capable de critiquer l'établissement et les leçons du texte qu'il annote. De surcroît, il était capable de longues séances de lecture suivie. En 1586 encore, il s'astreignait à lire d'une traite, en latin, les œuvres de Tacite, à la suggestion de son protecteur et ami, Louis de Foix ${ }^{38}$. Cette affirmation ainsi n'est pas à prendre au pied de la lettre. Toutefois, on ne saurait la réduire à l'expression factice d'une pose définissant un éthos aristocratique. Comme souvent chez Montaigne, le discours personnel est chargé d'une intention, il repose sur l'ironie, mais il porte sa réalité, inscrite dans le temps. Quelques années après avoir lu Tacite, au moment des derniers ajouts portés sur les Essais, Montaigne avait perdu cet ami, il était privé de sa conversation et de ses conseils. Les livres, qui jusqu'alors le liaient à des amis réels, ne suscitaient plus une même curiosité de sa part ni la même attention. Les propos désabusés du lecteur qui se borne à feuilleter des livres en passant ne sont pas en contradiction avec d'autres passages des Essais; ils sont l'expression même du temps qui passe et du changement à l'œuvre dans la longue durée du livre, de la rédaction des premiers chapitres, vers 1572, aux derniers ajouts portés sur la «copie d'auteur», dans les semaines précédant la mort de Montaigne, en septembre 1592. Les lectures et les modes de lecture reflètent trois phases de la vie même de l'écrivain, le temps de l'étude, le temps de l'action, le temps de la solitude. Les livres dont il parle dans la dernière rédaction du chapitre «De trois commerces» participent à une conversation indifférente; il les considère comme inutiles voire même néfastes, dans la mesure où ils offrent à l'instar des autres conversations courantes «un plaisir qui n'est pas net et pur» et qui n'apporte aucun soulagement au corps malade. Au milieu de ses livres, dans sa bibliothèque, Montaigne ne se soucie plus des livres, il n'entretient avec eux, en fait, aucun commerce suivi, il ne leur parle pas ou plus exactement, il ne leur parle plus. Ce sont des livres sans titres et sans auteurs, au contraire de ce qu'ils étaient pour lui, quinze ans plus tôt, alors qu'il rédigeait le chapitre qui leur était dévolu.

Ce serait donc une erreur que de considérer le dernier «commerce» avec les

(34) Voir A. Legros, La main grecque de Montaigne, «Bibliothèque d'humanisme et Renaissance», 61, 2, 1999, pp. 462-478.

(35) Voir M. SCREeCH, Montaigne's Annotated Copy of Lucretius, Genève, Droz, 1998. L'ensemble des «Notes de lecture» connues de Montaigne a été édité par A. Legros, Les Essais, éd. cit., pp. 1185 1310.
(36) Trois livres, sur la centaine de volumes conservés ayant appartenu à Montaigne portent la mention «Michaelis Montani et amicorum»; voir G. De BotTon et Fr. PotTiÉE-Sperry, À la recherche de la 'librairie' de Montaigne, «Bulletin du Bibliophile», 1997, 2, pp. 254-297.

(37) Les Essais, III, 3, p. 869.

(38) Ibid., III, 5, p. 986. 
livres, que Montaigne situe dans sa bibliothèque, comme une véritable conversation avec les grands auteurs du passé. Montaigne en fait n'évoque plus une telle relation fondée sur l'échange de la parole. Ce qu'il écrit de sa relation aux livres est à cet égard très différent et dans son ton et dans ses implications de ce qu'écrivait Machiavel, dans une lettre souvent mentionnée, adressée à son protecteur Francesco Vettori, en date du 10 décembre 1513. Le secrétaire florentin évoque les journées qu'il passe dans sa maison des champs, à Sant' Andrea in Percussino, à trente kilomètres de Florence, en une retraite volontaire, liée à ses difficultés financières. Il déplore la vacuité des journées passées dans la «pouillerie», où il est réduit à fréquenter des hommes du peuple, et célèbre au contraire la tout autre dignité de ses soirées:

Venuta la sera, mi ritorno in casa, et entro nel mio scrittoio: et in su l'uscio mi spoglio quella vesta cotidiana, piena di fango e di loto, e mi metto panni reali e curiali; e rivestito condecentemente entro nelle antiche corti degli antichi uomini, dove, da loro ricevuto amorevolmente, mi pasco di quel cibo, che solum è mio, e che io nacqui per lui; dove non mi vergogno parlare con loro, e domandarli delle ragioni delle loro azioni: e quelli per loro umanità mi rispondono, e non sento per quattro ore di tempo alcuna noia, sdimentico ogni affanno, non temo la povertà, non mi sbigottisce la morte ${ }^{39}$.

Il ne s'agit pas seulement, pour Machiavel, d'une lecture ou du moment privilégié de la lecture, après des occupations moins nobles, mais d'une cérémonie. Car la journée banale est aussi entrecoupée de moments de lecture. Machiavel lit entre le bois et l'auberge, près de sa volière:

Ho un libro sotto, o Dante o Petrarca, o un di questi poeti minori, come Tibullo, Ovidio e simili: leggo quelle loro amorose passioni e quelli loro amori, ricordomi de' mia, godomi un pezzo in questo pensiero.

Durant sa journée, Machiavel lit des livres qui appartiennent à un genre particulier, des recueils de poésie lyrique, dont il nomme les auteurs, italiens et latins. Le soir, il ne lit pas, ou du moins il fait plus que lire, et l'acte physique de la lecture n'est qu'une étape préliminaire et une médiation; ce n'est plus une lecture présentée comme telle, mais une véritable conversation, non pas avec des livres ni avec des auteurs précis, Tite-Live, Tacite ou Thucydide, mais avec les Anciens, avec toute la «cour des Anciens», dans une relation immédiate. Et dans cette conversation, c'est à Brutus et à Caton qu'il s'adresse et ceux-ci lui répondent.

Dans un cas, sous une forme familière, dans un cadre champêtre, la lecture suscite le souvenir, prétexte heureux à l'évocation de la jeunesse et des amours passés; elle est un passe-temps qui recrée, elle permet le retour au passé immédiat du lecteur, elle l'aide à vaincre la nostalgie. Par elle, Machiavel, en s'ouvrant aux livres, rentre en soi-même. Dans le second cas, la lecture ouvre un rituel aulique, marqué par le changement de vêtement et de langue, sans rien de parodique ou de factice, dont la puissance est véritablement magique à la manière d'une incantation: la lecture permet d'abolir la durée historique des siècles; les fantômes des grands hommes se révèlent, ils expliquent les mobiles de leurs actions passées, ils font comprendre les

(39) Machiavel, Lettre à Francesco Vettori, Opere, éd. C. Vivanti, Turin, Einaudi, 1999, t. II, pp. 294-297. Sur la mise en perspective historique de ce texte dans la tradition italienne, voir L. BoLzoNI, 'Entro nelle antique corti degli antiqui buomini': la lettura come incontro e dialogo con l'autore République des Lettres, République des Arts. Mélanges en l'bonneur de Marc Fumaroli, éd. C. Nativel et C. Mouchel, Genève, Droz, 2008, pp. 37-58.

(40) Voir D. ThORTON, The Scholar in bis Study. 
secrets de l'histoire. Rentrant chez lui, dans le secret de son studiolo improvisé, qui n'est pas même une véritable librairie, Machiavel sort de lui-même, sort de son temps propre et de son époque; le temps se dilate pour lui, non pas comme le temps trop long de la solitude, mais comme un temps plus plein, qui conduit à l'oubli de soi en tant qu'être soumis aux circonstances et aux malheurs humains, ennui, tourments, pauvreté, crainte de la mort. La conversation des livres, c'est-à-dire des Anciens et non pas seulement des auteurs anciens, est pour Machiavel une libération de l'âme, et son lieu propre, la bibliothèque, d'abord simple refugium animi, devient ainsi le lieu où s'opère une guérison intérieure, en une forme d'eutrapélie, de victoire sur la mélancolie et d'ouverture au monde. Cette conversation revigorante permet le retour à la conscience civile en même temps que la leçon donnée par les Anciens devient à son tour la matière d'un livre, Il Principe, les Discorsi, qui permettra de comprendre le présent. Sous cette forme, la lettre de Machiavel constitue un des textes majeurs de l'humanisme et de la culture européenne. Elle définit une relation vivante au passé, fondée sur les livres, mais dans l'oubli des livres en tant que médiation, une relation de parole, une conversation qui se fait écriture. Elle définit son rituel et sa symbolique, qui permettent la magie d'une double métamorphose: celle d'une solitude en présence vivante, celle d'une conversation en livre. Avec ce texte se définit aussi le lieu qui permet d'accueillir, sous forme réelle ou imaginaire, toute conversation civile et savante. Ce lieu prend ainsi une figure, au moment même, au début du XVI siècle, où se fait lieu, en termes concrets, inscrits dans l'architecture civile, le passage du studiolo à la bibliothèque, porteurs l'un et l'autre d'une fonction et d'une symbolique propres $^{40}$.

Rien de tel en revanche chez Montaigne, ou du moins plus rien de tel dans la dernière réaction du chapitre «De trois commerces». Alors que les Essais ne cessent de témoigner de l'omniprésence de l'Antiquité et de ses grands hommes jusque dans les ultimes rédactions, la bibliothèque n'est plus le lieu de leur évocation et les livres ne servent plus à les appeler auprès d'un Montaigne malade et vieilli. Les livres ne sont plus lus, mais simplement feuilletés, comme s'ils risquaient de détourner leur ancien lecteur d'un «commerce» plus essentiel. Dans les deux premiers livres, et dans la première rédaction du livre III, publiée en 1588, Montaigne opposait déjà la lecture à la conversation, suivant une opposition topique dans la culture aristocratique, sur laquelle reposera la célébration de «l'honnêteté» à l'âge classique: une heure d'entretien choisi vaut mieux que la lecture des livres les plus savants. Dans le chapitre «De l'art de conférer», Montaigne confirmait sa préférence pour la «conférence»:

L'estude des livres, c'est un mouvement languissant et foible qui n'eschauffe poinct: là où la conférence apprend et exerce en un coup ${ }^{41}$.

Dans ce texte toutefois, cette opposition joue sur un plan plus limité. Montaigne considère le livre comme un moyen d'étude, et cette limitation du rôle qu'il lui attribue se rattache à un argument d'ordre pédagogique, longuement développé dans le chapitre «De l'institution des enfans»: le livre apparaît moins efficace pour enseigner que la parole vive du maître, que l'échange et le dialogue ${ }^{42}$. Il convient ainsi de ne pas attribuer au terme de «conférence» une portée générale, d'en faire un synonyme

Ownership and Experience in Renaissance Italy, New Haven-London, Yale University Press, 1997, en particulier pp. 116-120.
(41) Les Essais, III, 8, p. 967.

(42) Voir M.-L. Demonet, Art de conférer, art de raisonner, «Cahiers Textuels», 1986, 2, pp. 18-29. 
de conversation ou d'entretien: la «conférence» n'est pour Montaigne qu'un mode particulier et en quelque sorte technique de la conversation.

Dans l'argumentation du chapitre «De trois commerces» en revanche, le «commerce» des livres et celui des hommes ne s'opposent pas mais se complètent. Les livres sont présentés comme le substitut des deux autres conversations. Ils sont toujours disponibles même s'ils offrent moins d'agréments. Leur commodité est l'objet d'un premier éloge, que vient précisément compléter la description de la librairie. Montaigne célèbre les livres en général, quels qu'ils soient et quel qu'en soit le sujet, en développant deux «lieux» rhétoriques complémentaires. Le premier, traditionnel et que Machiavel avait déjà évoqué, est celui du livre consolateur, du livre remède à la mélancolie:

Il me console en la vieillesse et en la solitude: il me decharge du poix d'une oisiveté ennuyeuse [...] il emousse les pointures de la douleur, si elle n'est point du tout extreme et maistresse ${ }^{43}$.

Le second argument topique de l'éloge est celui du livre servant de passe-temps. Montaigne met en évidence la portée de cette conception: «Si quelqu'un me dit, que c'est avilir les muses, de s'en servir seulement pour jouet». En ne les prenant que pour se divertir, pour détourner de soi le chagrin et l'ennui, il ôte aux livres leur importance lettrée et leur prestige savant.

Stefano Guazzo situait dans une bibliothèque la conversation privée entre le cavaliere et le médecin qu'il transcrivait dans sa Civil conversazione et qui en constituait la matière. Cette conversation avait une fin thérapeutique et devait permettre la guérison de la mélancolie dont était affecté l'homme de cour: «nelle picciole e rimote stanze dove io soglio tener riposti più per ornamento che per studio alcuni pochi libri» ${ }^{44}$. En associant lui aussi la conversation et la bibliothèque, Montaigne témoigne de sa connaissance du traité de Guazzo, auquel il fait une implicite référence. Mais la bibliothèque, sa bibliothèque à laquelle il consacre un si ample développement, n'est plus destinée à accueillir une civile conversation à la française. Elle sert de substitut à celle-ci, de même que les peintures érotiques du cabinet qui la jouxte, lui rappellent un temps qui n'est plus et dont le souvenir rend plus aiguës encore les souffrances de la maladie et de la vieillesse. Quelle autre conversation peut donc trouver sa place dans cette libraire édifiée en un pays «sauvage», que Montaigne, en un vigoureux contraste, situe loin de toute société civile, une librairie vouée au souvenir des conversations passées avec l'ami disparu, avec les Dames aimées ou désirées, au milieu de livres, lus jadis et désormais ennuyeux? Ces conditions de déshérence ne lui offraient plus qu'une conversation avec soi-même. Tout l'effort de Montaigne contre l'aliénation de la solitude, dont, vingt ans plus tôt, il avait mis en évidence le danger dans un des premiers chapitres des Essais, était d'en faire une véritable conversation. Mais à la différence de la situation qu'il avait connue au moment de sa retraite du Parlement, cette conversation avec soi-même était plus aisée. Elle pouvait prendre la forme d'une conversation avec un livre, mais un seul, celui qu'il ne cessait de lire, de relire mais aussi d'écrire et d'enrichir, ces Essais qu'il «enregistre et dicte», qu'il écrit comme il leur parle. Contre la langue même de Montaigne, on a interprété cette expression comme un redoublement synonymique, pour vouloir prouver que Montaigne incarnait la figure de l'écrivain au sens moderne du terme, qu'il s'était enfin libéré de la dépendance archaïque et socialement blâmable du secrétaire, et du même coup, pour

(43) Les Essais, cit., p. 868.

(44) S. Guazzo, La civil conversazione [1574], éd. A. Quondam, Ferrara, Panini Editore, 1993, t. I, p. 14. 
confirmer que l'Exemplaire de Bordeaux, un autographe, était le seul texte authentique $^{45}$. L'expression «enregistre et dicte» illustre en fait, avec une précision quasi technique, la préparation même du livre, selon les habitudes éditoriales de l'époque. Les deux termes ne sont ni antinomique ni synonymes, mais complémentaires; ils correspondent à deux étapes d'une même tâche. Montaigne «enregistre» ses derniers ajouts en les rédigeant lui-même dans les marges de ses exemplaires de travail, ainsi que l'atteste l'Exemplaire de Bordeaux; il en «dicte» la transcription à un secrétaire, sur une copie d'auteur, qu'il relira, corrigera et sur laquelle il reviendra porter lui-même d'ultimes modifications. La conversation avec le livre était aussi une conversation avec le secrétaire mais celle-ci, pour être moins univoque qu'il n'y paraît, n'était en rien une conversation civile.

Plusieurs années auparavant, les Essais avaient eu pour origine des conversations amicales dans la librairie, ainsi que Montaigne le rappelle à plusieurs reprises. Le chapitre «De l'institution des enfans» était né des suggestions d'un visiteur anonyme qui avait eu la primeur en manuscrit du chapitre «Du pédantisme»:

Quelcun doncq' ayant veu l'article precedant, me disoit chez moy l'autre jour, que je me devoys estre un peu estendu sur le discours de l'institution des enfans ${ }^{46}$.

La vicomtesse de Duras elle aussi était venue rendre visite à Montaigne en ce lieu. Il rédigeait alors le chapitre «de la ressemblance des enfans aux pères» qu'il lui dédia, en le rattachant précisément à leurs entretiens: «Vous y recognoistrez ce mesme port, et ce mesme air, que vous avez veu en sa conversation ${ }^{47}$. Douze ans plus tard, les Essais se poursuivaient seuls, désormais, comme la seule forme de conversation qui restait à Montaigne, mais aussi la seule qui fût digne de la «vacation» d'une grande âme confrontée à la solitude. Ils s'accomplissaient désormais hors de la bibliothèque, dans la «boutique de libraire», suscitant une autre forme de conversation, avec leurs premiers lecteurs $^{48}$.

JEAN BALSAMO

(45) Dans les Essais, «dicter» a bien son sens courant, ainsi que le confirment les trois autres emplois du verbe («dictée mot à mot par la bouche de Dieu», I, 56, p. 335, «des sainctes et divines chansons, que le Sainct Esprit a dicté en David», ibid., p. 338; «une lettre qu'il dicta», II, 16, p. 657), contrairement à l'affirmation de F. GARAVINI, Sur deux phrases des 'Essais' Etudes montaignistes en hommage à Pierre Michel, Paris, H. Champion, 1984, pp. 113-115. Le rôle du secrétaire, présent durant toute la rédaction du livre, a été mise en évi- dence par G. Hoffmann, Montaigne's Career, Oxford, Clarendon Press, 1998, tr. fr., Paris, H. Champion, 2009, pp. 53-78. Sur la procédure éditoriale suivie lors de la publication posthume, voir notre Introduction, Les Essais, cit., pp. xxxii-lv.

(46) Les Essais, I, 25, p. 153.

(47) Ibid., II, 37, p. 823.

(48) Voir, sur les premiers, notre étude: "Les Essais" de Montaigne et leurs premiers lecteurs: exemplaires annotés (1580-1598), «Montaigne Studies», XVI, 2004, p. 143-151. 